Mens

Revue d'histoire intellectuelle et culturelle

mens

\title{
Sur le romantisme littéraire canadien entre les années 1830 et 1860 : une étude comparative des discours sur la littérature de Joseph-Sabin Raymond et d'Henri-Raymond Casgrain
}

\section{Liliana Rizzuto}

Volume 19, numéro 1-2, automne 2018, printemps 2019

Les années 1840 : rupture ou réarticulation des possibles ?

URI : https://id.erudit.org/iderudit/1070072ar

DOI : https://doi.org/10.7202/1070072ar

Aller au sommaire du numéro

Éditeur(s)

Centre de recherche en civilisation canadienne-française

ISSN

1492-8647 (imprimé)

1927-9299 (numérique)

Découvrir la revue

Citer cet article

Rizzuto, L. (2018). Sur le romantisme littéraire canadien entre les années 1830 et 1860 : une étude comparative des discours sur la littérature de Joseph-Sabin Raymond et d'Henri-Raymond Casgrain. Mens, 19(1-2), 139-163.

https://doi.org/10.7202/1070072ar
Résumé de l'article

Le présent article propose une analyse du discours sur la littérature de Joseph-Sabin Raymond et d'Henri-Raymond Casgrain, deux des représentants les plus éminents du romantisme littéraire canadien de 1830 à 1860. Leurs points de vue différents, flagrants notamment dans les textes qu'ils publient respectivement dans le Foyer canadien au cours de l'année 1866, offrent l'occasion d'une comparaison éclairante sur deux définitions concurrentes de la littérature canadienne pendant la période : la première plus classique, abreuvée à même les traités d'éloquence antiques et le romantisme catholique français ; la seconde plus moderne, résolument nationaliste, plus conservatrice également, quoique toujours aussi romantique, même s'il ne s'agit peut-être que d'un "romantisme de façade ", ainsi que le suggère, entre autres, Micheline Cambron. 


\title{
Sur le romantisme littéraire canadien entre les années 1830 et 1860 : une étude comparative des discours sur la littérature de Joseph-Sabin Raymond et d'Henri-Raymond Casgrain
}

\author{
Liliana Rizzuto \\ Université Laval
}

\section{Résumé}

Le présent article propose une analyse du discours sur la littérature de Joseph-Sabin Raymond et d'Henri-Raymond Casgrain, deux des représentants les plus éminents du romantisme littéraire canadien de 1830 à 1860 . Leurs points de vue différents, flagrants notamment dans les textes qu'ils publient respectivement dans le Foyer canadien au cours de l'année 1866, offrent l'occasion d'une comparaison éclairante sur deux définitions concurrentes de la littérature canadienne pendant la période: la première plus classique, abreuvée à même les traités d'éloquence antiques et le romantisme catholique français; la seconde plus moderne, résolument nationaliste, plus conservatrice également, quoique toujours aussi romantique, même s'il ne s'agit peut-être que d'un "romantisme de façade", ainsi que le suggère, entre autres, Micheline Cambron.

\section{Abstract}

This article analyzes the discourse on literature by Joseph-Sabin Raymond and Henri-Raymond Casgrain, two of the most eminent representatives of Canadian literary romanticism from 1830 to 1860. Their different points of view offer the opportunity for an enlightening comparison on 
two competing definitions of Canadian literature during the period: the first approach, influenced by Roman rhetoric and French Catholic romanticism; the second, more modern, nationalist, conservative, still romantic, even if it is a "romantic façade" (Micheline Cambron).

Après les rébellions de 1837-1838 et l'adoption de l'Acte d'Union en 1840 , une période d'accalmie prolongée remplace l'effervescence des conflits armés. Selon les tenants de l'École littéraire de Québec, Henri-Raymond Casgrain en tête, c'est le moment de prendre un peu de recul. Pour citer l'incipit de son fameux article «Le mouvement littéraire en Canada» (1866): "Après deux siècles de luttes incessantes, de combats sans relâche, des jours plus calmes sont venus, et ont offert aux esprits ce recueillement indispensable au développement de la pensée ${ }^{1}$."

Sans plus d'ambages, le texte de l'abbé tire un trait sur tout un pan de l'histoire nationale, cette époque incertaine qui, depuis trop longtemps déjà, restreint et retarde l'essor d'une culture proprement canadienne-française. Son propos, romantique par bien des aspects, touche aux grandes topiques du siècle (l'histoire, la religion, le patriotisme, le progrès), mais il rejette également toute prise de position idéologique des littéraires, toute notion d'engagement ou de lutte à finir. La paix sociale devient une condition nécessaire à cette ère de prospérité qu'il voit s'affirmer pour la jeunesse canadienne depuis les travaux pionniers de Garneau et de Crémazie, considérés «à la fois comme ses ancêtres et ses contemporains ${ }^{2}$ ». Pour l'ecclésiastique né en 1831, tout commence avec eux, les premiers écrivains nationaux du pays, et il y a finalement peu à dire sur ce qui précède l'Histoire $d u$ Canada (1845-1852) et «Le vieux soldat canadien» (1855), avant le dénouement politique qui leur donne, comme à l'ensemble du

\footnotetext{
1 Henri-Raymond Casgrain, «Le mouvement littéraire en Canada», dans Le Foyer canadien: recueil littéraire et historique, t. IV, Québec, Bureaux du Foyer canadien, 1866, p. 2. Désormais, les références à ce texte seront indiquées par le sigle $M L C$, suivi du folio.

2 MLC, p. 3.
} 
peuple, une cause nouvelle de célébration. Cette position de l'auteur, emblématique du mouvement des années 1860, s'impose comme le fondement du romantisme institutionnalisé décrit, entre autres, par David M. Hayne ${ }^{3}$ et Manon Brunet ${ }^{4}$. Ainsi que le spécifie cette dernière, plusieurs textes similaires paraissent en 1866, tous signés par des hommes moins âgés que Casgrain lui-même ${ }^{5}$, dont "L'homme de lettres: sa mission dans la société moderne» de Faucher de SaintMaurice, "On Canadian Literature» d'Hector Fabre et «La poésie» de Félix-Gabriel Marchand ${ }^{6}$, qui confirment tous cette tendance du romantisme au Canada. Au cours de la même année, un autre article paru dans le Foyer canadien propose pourtant une perspective nettement différente. Le «Discours sur l'importance des études classiques ${ }^{7}$ " de Joseph-Sabin Raymond traite, lui aussi, du développement d'une littérature nationale, pieuse et patriotique, cependant, plutôt que d'en souligner l'originalité ou la nouveauté apparente, il évoque la continuité de son histoire grâce au collège classique et à ses racines grecques, latines et françaises. Davantage représentative de ce que l'on a appelé le "préromantisme canadien ${ }^{8}$ ", l'allocution

3 David M. Hayne, "Sur les traces du préromantisme canadien", dans Paul Wyczynski, Bernard Julien et Jean Ménard (dir.), Mouvement littéraire de Québec, 1860: bilan littéraire de l'année 1960, Ottawa, Éditions de l'Université d'Ottawa, 1961, p. 7-27, coll. «Archives des lettres canadiennes», t. I.

${ }^{4}$ Manon Brunet, «Mensonge et vérité romantiques: l'institutionnalisation du romantisme au XIX ${ }^{\mathrm{e}}$ siècle québécois», dans Maurice Lemire (dir.), Le romantisme au Canada, Québec, Nuit blanche éditeur, 1993, p. 136-154.

5 Félix-Gabriel Marchand, Hector Fabre et Narcisse-Henri-Édouard Faucher de Saint-Maurice sont nés en 1832, en 1834 et en 1844 respectivement.

6 Voir Brunet, «Mensonge et vérité romantiques», p. 139. Mentionnons au passage que ces trois écrivains sont également de fréquents collaborateurs du Foyer canadien.

7 Joseph-Sabin Raymond, «Discours sur l'importance des études classiques», dans Le Foyer canadien: recueil littéraire et historique, t. IV, Québec, Bureaux du Foyer canadien, 1866, p. 95-120 et 137-164. Désormais, les références à ce texte seront indiquées par le sigle $D E C$, suivi du folio.

${ }^{8}$ Manon Brunet préfere cette expression de Hayne à celle de Séraphin Marion, "le romantisme qui s'ignore», afin de désigner le romantisme au Canada avant 1860. Voir Brunet, «Mensonge et vérité romantiques», p. 136. 
du professeur, de vingt et un ans l'aîné de Casgrain, exprime le point de vue d'une ancienne génération de gens de lettres, celle qui a placé Montalembert ${ }^{9}$ et Lamartine devant Garneau et Crémazie, vécu la grandeur des patriotes avant d'en commémorer la disparition et, surtout, avant d'en lire le compte rendu modulé par les derniers essais nationalistes.

Il est entendu que le romantisme canadien, avant et après la date charnière de 1840 , ne peut être compris de manière monolithique, comme un ensemble cohérent, réglé et fortement ancré dans la tradition, à l'instar du classicisme dont il se distingue ainsi explicitement. Toute tentative de définition à partir de quelques textes, même les plus caractéristiques de la période, donnerait au mieux une vision incomplète d'un phénomène et d'un terme dont le sens et la portée ont largement fluctué tout au long du XIX ${ }^{e}$ siècle. Il reste que les articles d'Henri-Raymond Casgrain et de JosephSabin Raymond, tels que rassemblés dans le dernier recueil du Foyer canadien, offrent une vitrine unique sur le processus d'autonomisation des lettres canadiennes et, notamment, sur les dissensions qui participent alors de la construction du champ. Le mouvement littéraire connait son heure de gloire vers 1860, autour de Casgrain et de ses consorts de Québec, toutefois l'œuvre de Raymond rappelle que les considérations qui animent le groupe existaient déjà au cours des années 1830, dans ses propres écrits et dans ceux de ses jeunes pairs, dont Joseph-Guillaume Barthe, Louis-Auguste Olivier ${ }^{10}$ et le précoce Pierre-Joseph-Olivier Chauveau ${ }^{11}$. En examinant de plus

9 Voir Robert Sylvain, «Le premier disciple canadien de Montalembert: l'abbé Joseph-Sabin Raymond (avec une lettre inédite)", Revue d'histoire de l'Amérique française, vol. 17, $\mathrm{n}^{\circ} 1$ (juin 1963), p. 93-103.

${ }^{10}$ Olivier est notamment connu pour son article «Essai sur la littérature du Canada», paru dans la Revue canadienne en 1845, dans lequel il défend l'importance de donner une "couleur locale» et un "caractère propre à notre littérature». À ce propos, voir Vincent Masse, "L'Amérindien "d'un autre âge" dans la littérature québécoise au XIxe siècle», Tangence, n 90 (été 2009), p. 107-133.

${ }^{11}$ Raymond est né en 1810, Barthe et Olivier, en 1816 et Chauveau, en 1820. Malgré son jeune âge, ce dernier participe lui aussi à l'essor du romantisme au 
près les textes de Casgrain et de Raymond, ce sont en fait deux écoles de pensée, deux définitions concurrentes de la littérature qui se révèlent: la première plus classique, nourrie à même les traités d'éloquence antiques et le romantisme catholique français, celui du Génie du christianisme (1802) de Chateaubriand, mais aussi de l'Avenir (1830-1831) de Lamennais; la seconde plus moderne, résolument nationaliste et plus conservatrice également, quoique toujours romantique, même s'il ne s'agit plus peut-être que d'un «romantisme de façade ${ }^{12}{ }^{\prime}$, ainsi que le suggère Micheline Cambron.

De nombreux chercheurs ont tenté d'expliquer le bouleversement de la doxa romantique au Canada entre les années 1830 et 1860, d'aucuns parlant d'un changement de régime historique ${ }^{13}$, de la fin d'un récit ${ }^{14}$, ou d'un processus d'institutionnalisation qui remplace le jeune romantisme par un romantisme officiel et national ${ }^{15}$. Dans leurs études respectives, plusieurs ont tenu compte de l'influence de la parole prononcée par Casgrain, d'autres encore de la pensée de Raymond, mais aucun des deux auteurs ensemble, observant dans les mots de l'un ce qui peut rester d'impensé ou d'imprécis dans ceux de l'autre. Le présent article propose de se recentrer sur l'argumentation développée par les deux hommes et de voir dans leurs écrits jusqu'à quel point le romantisme canadien change et se transforme d'une génération à l'autre; comment leurs textes, pourtant publiés dans le même périodique et conçus

cours des années 1830, signant notamment les poèmes «L'insurrection» (1838) et "Adieux à Sir John Colborne» (1839) dans le journal Le Canadien. Voir, entre autres, Lucie Robert, "Autour de "L'insurrection” (1838) du jeune Chauveau", dans Bernard Andrès et Marc André Bernier (dir.), Portrait des arts, des lettres et de l'éloquence au Québec (1760-1840), Québec, Presses de l'Université Laval, 2002, p. 327-346.

12 Micheline Cambron, «Apothéose et fin du récit romantique au Québec», dans Lemire (dir.), Le romantisme au Canada, p. 155-175.

${ }^{13}$ Marie-Frédérique Desbiens, La plume pour épée: le premier romantisme canadien (1830-1860), thèse de doctorat (littératures française et québécoise), Québec, Université Laval, 2005.

${ }^{14}$ Cambron, «Apothéose et fin du récit romantique au Québec», p. 155-175.

${ }^{15}$ Brunet, «Mensonge et vérité romantiques», p. 136-154. 
pour le même auditoire, parviennent à présenter des positions si distinctes sur le plan de l'articulation rhétorique, et néanmoins complémentaires sur le fond, sur la défense de ce projet qu'ils promeuvent pareillement au nom de la communauté canadienne. À côté de ces deux écrits, il y a encore une série d'œuvres connexes, dont une en particulier remontant aux premiers essais littéraires de Joseph-Sabin Raymond, ses Entretiens sur l'éloquence et la littérature (1832-1834) ${ }^{16}$, qui montrent bien non seulement la stabilité du discours du professeur, mais aussi les mutations qu'il subit à la suite du relâchement des tensions soulignées par Casgrain. Grâce à ces sources diverses, trois points de comparaison seront examinés: le rapport au temps historique, la pensée religieuse et sociale, et le message adressé à la jeunesse.

\section{Le présent romantique et le rapport au passé national}

Anciens élèves du cours classique, tous deux ecclésiastiques, conférenciers et collaborateurs assidus des journaux, Casgrain et Raymond fréquentent les mêmes cercles et se connaissent déjà depuis un certain temps lorsque paraissent leurs articles dans les pages du Foyer canadien. Les hommes de lettres entretiennent d'ailleurs une brève correspondance vers la fin de l'année 1865, après que l'abbé a remarqué les derniers discours de son aîné et lui a envoyé ses félicitations pour un texte d'éloge sur l'encyclique Quanta cura, paru dans la Revue canadienne ${ }^{17}$. À l'époque, Casgrain ne bénéficie pas encore de la renommée sociale qui s'attache à son nom et au mouvement dont on lui attribue la paternité symbolique. Auteur de deux

${ }^{16}$ Joseph-Sabin Raymond, Entretiens sur l'éloquence et la littérature, édition critique de Marc André Bernier et de Marie Lise Laquerre, Québec, Presses de l'Université Laval, 2012.

${ }^{17}$ Voir la lettre datée du 4 décembre 1865, Archives du Séminaire de SaintHyacinthe, Fonds du Séminaire, Correspondance des Supérieurs, ASE7/13, $\mathrm{n}^{\circ} 22$, citée par Manon Brunet, dans «Les réseaux gaumistes constitutifs du réseau littéraire québécois du xixe siècle», Globe: revue internationale d'études québécoises, vol. 7, n 1 (2004), p. 168-169. 
ouvrages, Légendes canadiennes (1861) et Histoire de la mère Marie de l'ncarnation $(1864)^{18}$, il se consacre à l'édition du Foyer canadien (1863-1866), une revue de littérature qu'il fonde avec quelques amis suivant leur défection commune d'une publication périodique similaire, les Soirées canadiennes (1861-1865). Sa toute première collaboration avec l'équipe refondue se concrétise, en 1866, avec la sortie du dernier et plus notable tome de la série dont il signe trois textes: la biographie «F.X. Garneau», la bibliographie «CEuvres de Champlain» et, bien sûr, l'article liminaire «Le mouvement littéraire en Canada». En outre, étant donné son rôle prépondérant au sein de la revue à ce moment, l'abbé sélectionne et ordonne probablement lui-même les textes qui forment l'ultime mouture du recueil, incluant l'article de Raymond, lequel réalise aussi sa première et, dans son cas, unique collaboration avec le périodique.

En accord avec la mission du Foyer canadien, "recueil historique et littéraire» dédié à la production indigène du pays, les deux auteurs proposent des réflexions originales sur l'histoire récente des lettres canadiennes, qu'ils expliquent et commentent chacun à leur manière. Les choses ont changé au cours des dernières années, et pour le mieux, de cela ils sont absolument convaincus. Néanmoins, leur rapport au temps diffère sur quelques aspects majeurs, principalement en ce qui concerne les grands combats qui seraient, ou non, finalement derrière eux. Alors que l'aîné veut mettre en garde les lecteurs contre les périls qui les guettent et rappeler de cette façon les meilleurs moyens de s'en prémunir, son cadet se concentre sur les occasions offertes par la quiétude actuelle et la sensibilité nouvelle des littérateurs pour le passé national.

En fait, malgré son attitude cavalière au sujet des «deux siècles de luttes incessantes", le jugement de Casgrain rejoint pour l'essentiel celui de la majorité de ses contemporains, comme lui peu encline à considérer la possibilité d'une révolution encore à venir. Reconnue

${ }^{18}$ En 1862, il publie également une biographie, intitulée Le chevalier Falardeau, sous le pseudonyme d'Eugène de Rives. 
pour sa tendance outrancière au conservatisme, au repli sur soi et sur les traditions qui, paradoxalement, sont celles que ses collègues et lui-même contribuent à établir, la position de l'écrivain porte à son extrême l'engouement existant pour la commémoration de l'histoire. Non sans raison donc, on a dit que se perd chez lui le sentiment d'urgence romantique, le "présent romantique» dont parle Cambron, c'est-à-dire la volonté et la capacité, surtout, d'être de son temps. Sans doute, l'auteur n'est pas un homme d'action et «il lui manquera toujours l'ancrage dans le présent qu'incarnaient encore en 1844-1845 les patriotes exilés ${ }^{19}$ ". Il demeure que le critique signe une œuvre unanimement qualifiée de "programmatique» et, en l'occurrence, initiatrice d'un mouvement dont il devient personnellement l'animateur énergique. Il y a dans son texte les marqueurs de l'impulsion qu'il observe dans les lettres canadiennes, des qualités novatrices, voire engagées, dont on serait en peine de ne pas signaler la correspondance avec les idéaux romantiques de l'époque.

Dès les premières pages de l'article, la posture de Casgrain convoque, en effet, l'assurance des grands manifestes du siècle, n'ayant de cesse de préciser que les circonstances sont différentes, que la conjoncture se prête désormais, et de manière idéale, aux légitimes ambitions littéraires des Canadiens. Les indices temporels abondent, et l'auteur insiste sur une coupure nette entre le temps d'avant, «l'époque de l'expansion physique», et maintenant, «l'époque de l'expansion intellectuelle»:

Si nous avons tardé longtemps à diriger notre attention vers la culture des lettres, c'est qu'après de faibles commencements, des guerres interminables, au lendemain des désastres de la conquête, nous avions tant de précieuses choses à sauver du naufrage! notre foi, notre langue, nos lois, toutes nos libertés, la patrie tout entière. Il y a lieu même de s'étonner des progrès qui ont été faits, malgré tant d'obstacles ${ }^{20}$.

\footnotetext{
${ }^{19}$ Cambron, «Apothéose et fin du récit romantique au Québec», p. 171.

${ }^{20}$ MLC, p. 24.
} 
Heureusement, les progrès se sont maintenus:

L'éducation s'est répandue rapidement: les sources intellectuelles ont été versées à flots sur la génération présente, tandis que l'horizon politique s'élargissait devant elle et donnait libre cours à toutes ses généreuses aspirations; et aujourd'hui l'on peut compter parmi nous toute une pléiade d'hommes lettrés, animés d'un noble enthousiasme, et qui s'occupent, avec ardeur, à exploiter nos vieilles chroniques et à célébrer nos gloires nationales ${ }^{21}$.

Lecclésiastique a des mots durs pour évoquer les événements entourant la Conquête. Préférant éviter de nommer précisément les épreuves, les sacrifices et les revirements divers qui composent cette période chaotique, il multiplie les images et les adjectifs suggestifs, opposant le «naufrage» du temps jadis aux «flots" des "sources intellectuelles» qui baignent actuellement la jeunesse. En dépit de l'antinomie postulée cependant, passé et présent tendent à se confondre au fur et à mesure qu'avance la démonstration. D'une phrase à l'autre, la conception du passé se module afin de suivre l'argumentation de l'auteur et révéler la marche du progrès qui explique et résout le problème du retard culturel du pays. Plus loin dans le texte, le souvenir des événements désastreux s'efface encore davantage au profit des récits fabuleux des ancêtres, symboles annonciateurs de la grandeur de la nation. Pour Casgrain, il ne s'agit que d'un juste retour du balancier; après tout, l'étude des temps anciens n'équivaut pas à fuir le présent, mais à l'actualiser, de façon à donner au peuple ce qui lui a toujours fait défaut, sa propre histoire.

Dans son rappel des contrariétés d'antan, le critique évoque également la génération des patriotes, à laquelle il réserve le même traitement téléologique. Éternel optimiste, l'abbé va d'ailleurs jusqu’à lui reconnaître le mérite d'avoir mené les "conquêtes politiques» nécessaires à l'«indépendance nationale ${ }^{22} »$. C'est connu, Casgrain

${ }^{21} M L C$, p. 2.

${ }^{22}$ Voici le passage en question: «L'ébranlement imprimé alors aux intelligences avait été merveilleusement secondé par ces conquêtes politiques. La génération 
aime à réécrire l'histoire, en particulier dans ses premiers textes ${ }^{23}$, et celui-ci ne fait pas exception. En l'occurrence, il ressort de sa lecture révisionniste des événements une volonté persistante de polir et de légitimer ce pan encore trop récent du passé canadien. Le simple fait d'éviter les mots d'échec et de défaite, pour parler plutôt de "l'horizon politique qui s'élargissait", paraît spécialement révélateur de sa position, tout comme sa manière de lier la triste fin des insurgés à l'explication de l'Histoire de François-Xavier Garneau, une œuvre «lumineuse» selon lui:

Ce livre était une révélation pour nous. Cette clarté lumineuse qui se levait tout à coup sur un sol vierge [...], les monuments et les souvenirs, nous ravissait d'étonnement autant que d'admiration.

Que de fois ne nous sommes-nous pas dits [sic], à l'aspect des larges perspectives qui s'ouvraient devant nous: - cette terre si belle, si luxuriante, est celle que nous foulons sous nos pieds, c'est le sol de la patrie! Avec quel noble orgueil, nous écoutions les divers chants de cette brillante épopée ${ }^{24}$ !

Le passé ne semble plus si sombre une fois illuminé. Sous ce nouvel éclairage, même le réel apparaît plus beau, plus riche si, du moins, l'écrivain, comme Garneau lui-même, parvient à faire ressortir ces qualités en dépit des événements eux-mêmes. "Les pieds ici, les yeux ailleurs", disait Hugo à propos du poète romantique. Pour Casgrain, il ne faut retenir de ces épisodes que ce qui convient à l'appréciation du présent, ce qui permet de voir la bravoure pardelà la défaite et, en priorité, la force inébranlable d'un peuple toujours vivant. C'est le rôle de la littérature et de l'histoire, comme genre littéraire, de rendre légitimes et signifiants les moments les

nouvelle, plongée dans cette atmosphère féconde [...] s'élançait avec amour dans l'étude, afin d'être prête, un jour, à remplir toutes les carrières que ce règne d'indépendance nationale ouvrait à ses légitimes ambitions.» $M L C$, p. 2-3.

${ }^{23}$ Maurice Lemire, "Henri-Raymond Casgrain, historien", Voix et images, n 65 (hiver 1997), p. 261-275.

${ }^{24} M L C$, p. 4. 
plus incompréhensibles et apparemment injustifiés du passé. Pour Joseph-Sabin Raymond, toutefois, n'en déplaise à Garneau, la leçon de l'Histoire peut parfois se révéler beaucoup moins «lumineuse».

Comme l'ont mentionné avec raison les nombreux lecteurs de l'œuvre du professeur de rhétorique, de Galarneau ${ }^{25}$ à Provost $^{26}$ et jusqu’à Bernier et Laquerre ${ }^{27}$, ses propos ne changent guère, entre les années 1830 et 1860 , en ce qui concerne sa conception de la littérature. Sans être anhistorique, son discours semble en fait quelque peu imperméable au passage du temps, en ce sens qu'il ne cherche pas tant à caractériser le présent par opposition au passé qu’à réfléchir aux perspectives d'avenir et à la préparation de ce futur, qu'il perçoit aussi plein de promesses. S’il est néanmoins une différence entre ses premiers écrits et le texte qui parait dans le Foyer canadien à côté de celui de Casgrain, c'est que la littérature seulement souhaitée et idéalement théorisée devient progressivement la littérature présente ou, à tout le moins, possible ici même au Canada. Un exemple de cette posture se retrouve dans l'un de ses premiers ouvrages de jeunesse, Entretiens sur l'éloquence et la littérature (1832-1834).

Volume resté longtemps inédit, mais dont le message rejoint nombre de ses publications ultérieures, les Entretiens sur l'éloquence et la littérature comprennent deux textes construits à la manière des Dialogues sur l'éloquence (1718) de Fénelon. Les interlocuteurs y discutent longuement de l'époque actuelle et des années troubles qui semblent devoir se poursuivre. Si, dans son texte, Casgrain parlait d'«obstacles» à l'avènement de la littérature, Raymond, lui, parle du «délire» qui la rend nécessaire. Le deviseur $\mathrm{D}$, en

${ }^{25}$ Claude Galarneau, «L'abbé Joseph-Sabin Raymond et les grands romantiques français (1834-1857)", Rapports annuels de la Société historique du Canada, vol. 42, n 1 (1963), p. 81-88.

${ }^{26}$ Guy Provost, "Conception de la littérature chez Joseph-Sabin Raymond", Revue d'histoire de l'Amérique française, vol. 32, $\mathrm{n}^{\circ} 4$ (mars 1979), p. 585-602.

${ }^{27}$ Marc André Bernier et Marie Lise Laquerre, «Préface. Aux origines d'une esthétique de l'exaltation", dans Raymond, Entretiens sur l'éloquence et la littérature, p. $5-21$. 
particulier, souligne le rôle important des lettres dans la résolution de ce conflit:

Aux jours que nos yeux viennent de voir s'écouler, le délire a troublé aussi la société, qui a méconnu les lois du régulateur suprême; pour peine de son crime, l'ordre, la tranquillité lui furent ravis [...]. Poètes, écrivains, il est temps: touchez la harpe, dites en accents harmonieux la beauté de la religion et vos sons calmeront le délire [...] et l'ordre et la paix et le bonheur aimeront encore à visiter la terre ${ }^{28}$.

La personne qui lui succède renchérit de plus belle:

Je le dis sans détour: le temps de la décadence est terminé, nous sommes à une époque de régénération [...], une régénération sociale faite par la religion: oui, une réaction puissante s'opère dans le monde social, forte du génie, de la science et du zèle de ceux qui travaillent à l'opérer. Et cette réaction, c'est aux lettres qu'on la doit $[\ldots]^{29}$.

D'emblée, la parole «réactionnaire» de Raymond contraste singulièrement avec celle de son homologue, et même avec celle de son texte plus tardif, écrit 30 ans plus tard. À cette époque, l'œuvre du jeune écrivain montre son adhésion aux thèses du renouveau religieux français ${ }^{30}$ et, notamment, à la théorie du sens commun de Lamennais. Selon ses propres observations, il n'y a de vérité qu'universelle, et la paix, le bonheur, même le talent dépendent entièrement de l'intervention du divin. La pensée du progrès dont parle Casgrain n'entre donc nullement dans cette équation. Il n'est pas question d'une insuffisance de ressources ou d'éducation, et encore moins des «obstacles» posés par la Conquête ou les remous sociaux et politiques qui s'en suivent. Ce qui manquait, et ce qui manque toujours au rayonnement des lettres canadiennes, ce sont les lumières de la religion ainsi qu'une soumission complète aux

\footnotetext{
${ }^{28}$ Raymond, Entretiens sur l'éloquence et la littérature, p. 91.

${ }^{29}$ Ibid., p. 91.

${ }^{30}$ Provost, «Conception de la littérature», p. 587.
} 
règles de l'Église. Qu'est-ce que le délire, en effet, sinon une perception erronée de la réalité et l'incapacité à différencier le vrai du faux, voire à se bercer volontairement d'idées fausses, mais plaisantes?

Dans son "Discours sur l'importance des études classiques", publié en 1866 dans le Foyer canadien, l'auteur reste campé sur ses positions et continue de professer sa confiance dans le "zèle» des hommes de bien. Vingt-cinq ans après l'Acte d'Union, il ne parle plus de "réaction" ni de "régénération", mais croit toujours à la nécessité d'un changement fondamental, à l'image de ce que proposaient les insurgés de 1837-1838. S’adressant aux élèves du séminaire de Saint-Hyacinthe dont il est le supérieur, Raymond évoque lui aussi les patriotes et souligne tout ce que la patrie doit à ces derniers, particulièrement à son beau-frère, Augustin-Norbert Morin, alors présent dans l'auditoire:

[S]i comme vous l'avez souvent entendu répéter: les exemples sont plus puissants que les leçons, je n'ai pas besoin d'insister davantage pour vous faire voir ce que de vastes connaissances, fruits d'une continuelle étude peuvent apporter à un citoyen pour l'illustration de son nom et l'importance de ses services à l'égard de la patrie; vous en avez une éclatante manifestation au milieu de vous ${ }^{31}$.

La retranscription de l'allocution prononcée en juin 1865 ne permet pas de savoir si Morin s'est levé ou si les gens rassemblés ont autrement reconnu l'importance de l'homme qui se trouvait parmi eux, ancien rédacteur des anciennes 92 résolutions. Sans doute peut-on affirmer que la présence du patriote a été soulignée et a donné une autre résonance aux assertions de son parent, soudainement moins théoriques et plus actuelles. Pour Raymond, au reste, la jeunesse

${ }^{31}$ Une note dans le Foyer canadien précise qu'il s'agit d'une «allusion à l'honorable A. N. Morin, présent à ces exercices littéraires». Il est à noter que Morin décède en juillet 1865, à l'âge de 61 ans, peu après la tenue de la cérémonie ( $D E C$, p. 159). 
du pays ne doit pas seulement célébrer les accomplissements de ses prédécesseurs, mais s'en inspirer.

Croit-on que si les collèges canadiens avaient manqué, la patrie serait ce qu'elle est aujourd'hui; que si ceux qui en ont défendu si heureusement les intérêts n'avaient eu qu'une éducation bornée puisée à des institutions inférieures, leur parole eut eu la même force, leur action la même habileté, leur patriotisme le même dévouement? Eh bien! la nationalité canadienne, elle a encore des dangers à courir, des questions vitales pour elle à discuter, de fortes luttes à soutenir. Elle vaincra si elle a des athlètes munis d'armes trempées à une solide éducation: qu'on me permette de le dire, les colléges $[s i c]$ sont les arsenaux de la patrie ${ }^{32}$.

Il y a une fierté et une déférence dans les paroles de Raymond qui font défaut à Casgrain dès qu'il se remémore la grandeur des patriotes. Le cadet veut lancer un "mouvement", une action citoyenne et engagée, mais c’est son aîné qui en pose les bases concrètes. Alors que le premier tente d'aiguiller une génération qu'il considère comme étant arrivée à un moment charnière de son histoire, à cette "époque de la littérature» qui lui permettra de recueillir les fruits du labeur de ses ancêtres, le second cherche plutôt à préparer une nouvelle armée de jeunes intellectuels qui, elle, ajoutera des pages à cette histoire.

\section{De l'Église et de la société}

Pour l'homme de lettres qui a connu les rébellions des années 1830, la paix actuelle reste toute relative. Il faut encore se préparer au combat, à la défense et à l'illustration de l'identité canadienne-française et, en premier lieu, de ce qui doit la définir au plus haut point: sa religion. Dans un autre texte intitulé «De l'Église et de l'État» (1866) publié dans la Revue canadienne, Raymond déclare comme Lamennais, "point d'ordre social possible sans la religion ${ }^{33}$ ». Sur ce

\footnotetext{
${ }^{32}$ DEC, p. 159.

${ }^{33}$ Joseph-Sabin Raymond, «De l'Église et de l'État», Revue canadienne, n 1 (janvier 1866), p. 46.
} 
sujet au moins, Casgrain est entièrement d'accord, même si pour lui la littérature nationale ne peut exister sans exprimer aussi, et même nécessairement, cette caractéristique du peuple canadien.

La conception des lettres du dernier apparaît nettement plus matérialiste que celle de son aîné. En fait, bien que l'abbé renie lui-même cette influence, son approche n'est pas sans rappeler celle du réalisme français et de sa fameuse théorie du reflet. Le passage $\mathrm{du}$ «Mouvement littéraire en Canada» concernant la littérature comme miroir est bien connu, et fort long d'ailleurs, mais il mérite d'être cité dans toute son étendue déclamatoire:

$\mathrm{Si}$, comme il est incontestable, la littérature est le reflet des mœurs, du caractère, des aptitudes, du génie d'une nation, si elle garde aussi l'empreinte des lieux d'où elle surgit, la nôtre sera grave, méditative, spiritualiste, religieuse, en même temps elle sera largement découpée comme nos vastes fleuves, mystérieuse comme les échos de nos immenses et impénétrables forêts, mélancolique comme nos pâles soirs d'automne, chaste et pure comme le manteau virginal de nos longs hivers.

Mais surtout elle sera essentiellement croyante, religieuse; elle sera sa forme caractéristique, son expression; sinon elle ne vivra pas, elle se tuera elle-même. C'est sa seule condition d'être $[\ldots]^{34}$.

À nouveau, il répète plus loin: "[S]a voie est tracée d'avance: elle sera le miroir fidèle de notre petit peuple ${ }^{35}$." Malgré l'influence lamennaisienne palpable ici, tout comme chez Raymond, Casgrain n'évoque pas le réenchantement du monde ni la beauté ou le génie du christianisme. Il n'y a pas le même mysticisme dans son écriture que dans celle de son homologue. La multiplication des comparaisons, enchaînées sommairement, pèse lourdement sur le lyrisme des mots employés et on peine à ressentir le mystère, la mélancolie, la pureté du paysage décrit. L'auteur, qui se montre généralement si prompt à faire entendre ses impressions personnelles, fait preuve

\footnotetext{
${ }^{34} M L C$, p. 26.

${ }^{35}$ MLC, p. 26.
} 
d'une objectivité étonnante par rapport à la majesté des lieux, présentés en fonction de leur grandeur pour les fleuves et les forêts, ou de leur couleur pour les soirées d'automne et les hivers.

Casgrain ne rend pas compte d'une littérature inspirée et inspirante, mais bien d'une littérature fidèle, qui traduit avec justesse la familiarité du réel. La différence entre l'opinion de son aîné et la sienne, remarquable sur ce point, est illustrée notamment dans un passage du "Discours sur l'importance des études classiques" mentionnant la pensée de Louis de Bonald et sa célèbre phrase: «La littérature est l'expression de la société».

[R]ien de plus juste que ce mot d'un noble génie. Mais ne peut-on pas dire aussi que la société en s'imprégnant de l'esprit des écrits qui sont lus partout avec avidité, met bientôt en pratique les principes et les sentiments qu'elle puise de toutes parts, et qu'elle se modèle sur les types que l'art lui a présentés, en sorte qu'elle devient pour ainsi dire, la réalisation de la littérature ${ }^{36}$.

Il y a dans cet extrait trop court d'une pensée développée sur plusieurs pages l'idée d'une relation dynamique entre la littérature et la société, «filles et compagnes» de l'Église, en plus d'une préoccupation toute chrétienne pour l'âme des fidèles. L'auteur veut une littérature qui soit avant tout une forme d'apologétique, qui élève les esprits et éloigne de l'impéritie. Il ne s'agit pas seulement de brosser le tableau des mours du peuple canadien, avec tous ses torts et ses travers, mais, plus significativement, de travailler à son émancipation sociale et morale. C'est bien là le mot d'ordre du romantisme, le premier romantisme de Lamartine et de Hugo, même si l'homme de lettres se montre lui-même très critique d'un livre comme Les misérables $^{37}$, trop réaliste peut-être, trop romanesque sans doute.

La rhétorique "émotionaliste» de Raymond, pour reprendre l'expression de Bernier et de Laquerre, l'amène à valoriser une poésie à fleur de peau, subjective et intime, l'œuvre d'un écrivain peu

\footnotetext{
${ }^{36}$ DEC, p. 115-116.

${ }^{37}$ DEC, p. 119.
} 
tenté par la gloire et sachant allier « la profondeur des pensées, l'éclat des images et la force de l'émotion ${ }^{38}$ ". Il exige de belles phrases, de beaux sentiments, pas un simple reflet de la société. Ce n'est pas non plus ce que désire Casgrain, bien sûr, pourtant lorsque ce dernier évoque le réalisme moderne, pour mieux le fustiger, il insiste sur l'idée que la littérature canadienne doit « faire aimer le bien » en évoquant les faits et le passé si cher aux Canadiens. Contrastant singulièrement avec l'enchaînement de plates comparaisons mentionné plus haut, ce passage de l'article déploie une hypotypose évocatrice, davantage caractéristique de l'écriture de l'abbé:

Heureusement que, jusqu'à ce jour, notre littérature a compris sa mission, celle de favoriser les saines doctrines, de faire aimer, admirer le beau, connaître le vrai, de moraliser le peuple en ouvrant son âme à tous les nobles sentiments, en murmurant à son oreille, avec les noms chers à ses souvenirs, les actions qui les ont rendus dignes de vivre, en couronnant leurs vertus de son auréole, en montrant du doigt les sentiers qui mènent à son immortalité 39 .

Si la première partie de cette phrase reprend l'essentiel du message de Raymond, qui est celui du jeune romantisme, la seconde partie, mentionnant les sentiers de la gloire, ramène le discours à des considérations historiques et nationales. Malgré tout, ce murmure à l'oreille du peuple personnalise la discussion, la rend plus humaine, plus sensible. Casgrain touche à l'éloquence chaque fois qu'il s'exprime sur le pays, ses souvenirs, son «immortalité». De fait, la gloire et l'admiration reviennent de manière récurrente dans la réflexion de l'écrivain, non pas spécifiquement à propos de l'Église, mais encore à propos de Garneau, de Crémazie, de la jeunesse canadienne. À travers eux, c'est la nation entière qu'il célèbre et encense avec émotion.

Alors que Raymond, à l'instar de Chateaubriand, révère les enseignements de Louis de Bonald, auteur et politicien

\footnotetext{
${ }^{38}$ Voir Bernier et Laquerre, «Préface», p. 17-18.

${ }^{39}$ MLC, p. 26-27.
} 
contre-révolutionnaire, Casgrain, lui, suit la pensée de FrançoisEdme Rameau de Saint-Père, historien et sociologue français né plus de 30 ans après 1789. Prenant exemple sur celui qui, depuis quelques années déjà, avait constaté l' "entière ignorance» dans laquelle le peuple canadien avait été "abandonné en 1760 ", il cite longuement un extrait de La France aux colonies (1859):

"Il ne nous semble point être dans la destiné [sic] du Canada», dit avec beaucoup de justesse M. Rameau, "d'être une nation industrielle ou commerciale [...]. [I]l appartient au Canada de s'approprier avec désintéressement et une noble fierté le côté intellectuel, scientifique et artistique du mouvement américain, en s'adonnant avec préférence au culte du sentiment, de la pensée et du beau ${ }^{40} »$.

Il ajoute ensuite:

Tel est aussi le partage réservé à la France américaine [...]. Et, certes, nous n'avons pas à nous en plaindre; car c'est en quelque sorte la meilleure part de l'Évangile, celle de la poétique Marie, par opposition à celle de Marthe l'affairée. L'infériorité du nombre et de la fortune n'empêche nullement de conquérir cette situation, qui tôt ou tard devient toujours la première ${ }^{41}$.

Soucieux de justifier ses propos en convoquant l'avis d'un expert, Casgrain fournit des explications «scientifiques" concernant la réussite prochaine du mouvement littéraire canadien. Les pensées rationnelle et religieuse travaillent ainsi de concert pour tracer la droite ligne vers la conquête des lettres nationales, celle qui doit conduire au rayonnement de la race, ici comme à l'étranger. Pour cela, la littérature n'a pas à changer le peuple, à l'émanciper ou à l'affranchir autrement d'un quelconque joug, elle doit simplement le révéler à lui-même, lui donner les moyens de ses ambitions afin qu'il prenne enfin sa place aux côtés des grandes nations.

${ }^{40}$ Cette citation est tirée de l'œuvre de François-Edme Rameau de Saint-Père, $L a$ France aux colonies, Paris, A. Jouby libraire-éditeur, 1859, p. 266.

${ }^{41} M L C$, p. 28. 


\section{Un message à la jeunesse}

Au-delà de leur divergence de points de vue, de leurs rapports au temps, à l'Église et à la société canadienne qui se rejoignent et s'entrechoquent par moments, Casgrain et Raymond se préoccupent tous deux de la jeunesse canadienne, de son avenir, de son éducation. Déjà devenus des hommes d'un certain âge, ils veulent conscientiser les jeunes hommes aux études ou récemment diplômés à l'importance de la lecture, de l'écriture et de la poursuite de l'avancement moral et intellectuel sous toutes ses formes. La connaissance de l'histoire, en tout premier lieu, doit être leur priorité, car, comme le souligne Raymond, elle n'est "pas seulement la connaissance du passé, elle est encore, jusqu'à un certain point, celle de l'avenir ${ }^{42}$ ». Ce futur prospère que l'un et l'autre garantissent aux générations suivantes differe sur plusieurs points, pour toutes les raisons déjà mentionnées, et cela, même si leurs deux visions convergent finalement sur l'essentiel. C'est la rhétorique du message, fondamentalement, qui révèle des antinomies patentes entre les deux écrivains.

Malgré le militantisme latent du discours de Raymond, a priori plus susceptible d'encourager l'esprit d'initiative chez ses pupilles, l'ancien professeur se montre de fait assez prudent dans ses propos concernant l'avenir de la relève canadienne. Spécifiquement, il veut dissuader tout comportement arriviste et faire comprendre aux jeunes qu'ils doivent entrer dans la vie active sans attente quant à leur gloire ou à leur renommée personnelle. En cette occasion solennelle de la cérémonie de remise des prix annuels au séminaire de Saint-Hyacinthe, il rappelle aux étudiants qu'ils ont été formés afin de servir Dieu, et donc la patrie:

Apprenez à servir la patrie avec dévouement et générosité, non par une ambition égoïste qui cherche l'honneur pour soi [...]. Servez la patrie pour elle-même, parce qu'étant votre mère, elle réclame avec droit les services dévoués de ses enfants, parce que

${ }^{42}$ DEC, p. 109. 
l'amour pour elle est une vertu morale inspirée par la raison et le plus noble instinct du cœur $[\ldots]^{43}$.

Cette entité abstraite, idéale et maternelle, évoquée par Raymond, impose des obligations bien différentes que celle que mentionne Casgrain dans son propre texte. Les Canadiens sont les enfants de la Patrie, ses humbles serviteurs, et leur affection pour elle relève de l'attachement émotif. Il n'y a pas de pareille gratitude chez Casgrain, car la Patrie, elle est ce que le peuple canadien en fait, son produit, l'expression concrète de ce que ses membres peuvent accomplir s'ils travaillent ensemble vers la réalisation d'un but commun. La notion de service public, absente dans "Le mouvement littéraire en Canada", donne au discours du recteur une gravité qui responsabilise les jeunes auxquels il s'adresse directement: "[A]pprenez à servir la patrie», «servez la patrie, répète-t-il, c'est à vous que revient cette tâche».

L'attente sociétale réactualisée dans l'enceinte du collège ne signifie pas pour autant que les étudiants doivent s'empresser de se lancer dans des carrières ou des initiatives citoyennes pour lesquelles ils n'ont pas les aptitudes nécessaires, ou qui ne répondent pas à un besoin réel. Aussi Raymond préconise-t-il le discernement à ceux qui voudraient embrasser le métier des lettres; trop nombreux sont déjà ceux qui le font mal et mal à propos:

On entend trop souvent une parole qui n'est qu'une vaine déclamation, sans aucune dignité, blessant même les règles élémentaires de la langue dont on se sert. Que d'écrits communiqués aux feuilles périodiques qui ne sont que l'expression de l'ignorance et de l'impéritie de leurs auteurs? Notre gloire nationale peut souffrir de cette parole orale ou écrite si peu digne d'attention et si peu propre à une influence salutaire. [...] Étudiez, remplissez vos esprits de connaissances, pénétrez-vous de la sagesse et du goût des bons modèles, et vous pourrez ensuite bien parler et bien écrire ${ }^{44}$.

\footnotetext{
${ }^{43}$ DEC, p. 99.

${ }^{44} D E C$, p. 149.
} 
Ces considérations classiques sur l'éloquence et la justesse de la parole n'étonnent guère de la part d'un maître de rhétorique. La critique concernant les écrits journalistiques, plus pointue, trahit toutefois une pensée conservatrice qui contraste avec le libéralisme social de l'auteur. Bien sûr, ce sont autant de considérations qui n'apparaissent à aucun endroit dans le discours de l'abbé Casgrain. Raymond n'est pas non plus un homme d'action, mais peut-être, sur cet aspect, l'est-il encore moins que son cadet. Il parle des collèges comme des "arsenaux de la patrie», mais recommande aux collégiens une retenue qui les éloigne du débat public et les prémunit contre tous ces essais et erreurs qui parsèment bien souvent les premières publications d'écrivains. En outre, il leur conseille de concentrer leurs efforts sur l'étude des modèles et de la grammaire. L'originalité et l'innovation restent importantes, mais pas au mépris des règles les plus élémentaires de l'harmonie et de la bonne compréhension.

Raymond s'intéresse d'abord aux qualités morales de la littérature, à sa justesse, à sa beauté. Il conçoit la jeune génération comme la gardienne d'un certain ordre social, celui qui a été établi par les prédécesseurs et le cours classique. Un tel respect des traditions ne rejoint pas son collègue. Sur ce point, Casgrain ne se satisfait d'aucune règle ni d'aucun modèle. Par son propre exemple, il montre qu'il vaut mieux provoquer les choses et donner soi-même l'impulsion de départ à un projet dont on ne sait pas s'il se réalisera un jour, que d'attendre qu'il se concrétise pour en discuter ensuite. C'est également le message qu'il veut transmettre à la jeunesse canadienne:

Ah! S'il nous était donné de nous adresser à la jeune génération qui voit l'avenir souriant lui tendre les bras, nous lui dirions avec l'accent de l'émotion : - Vous avez devant vous une des plus magnifiques carrières qu'il soit donné à des hommes d'ambitionner. Vous avez dans l'âme et sous les yeux toutes les sources d'inspiration. Vous pouvez, si vous savez exploiter ces ressources inépuisables, créer des œuvres d'intelligence qui s'imposeront à l'admiration. D'une main saisissant les trésors du passé, de l'autre ceux de l'avenir, et les réunissant aux richesses du présent, 
vous élèverez un édifice qui sera, avec la religion, le plus ferme rempart de la nationalité canadienne ${ }^{45}$.

L'auteur envisage le mouvement littéraire canadien avec une confiance immodérée. L' «avenir souriant» tend les bras à la jeunesse canadienne, et cette dernière ne doit pas craindre l'incorrection stylistique ni les erreurs factuelles. Au contraire, elle n'a qu'à parler avec son cœur, avec ce qu'elle a "dans l'âme et sous les yeux». Le Canada possède cet avantage d'être un pays jeune: il n'y a pas de canons, pas de règles à suivre, et son patrimoine littéraire, c'est lui qui l'invente.

\section{Deux points de vue non pas contraires, mais contrastés}

Il y aurait encore tant à dire sur ces deux textes, sur le romantisme toujours plein de classicisme chez Raymond et tendant déjà vers une certaine forme de réalisme chez Casgrain, sur leurs opinions concernant la critique littéraire, la fiction, le roman. Sans surprise, dans l'ensemble, les positions des deux hommes paraissent très similaires. Les articles qu'ils publient respectivement dans le Foyer canadien en 1866 promeuvent avec un même enthousiasme l'avancement des lettres nationales, un art sain et éloquent, portant fièrement les couleurs locales. Bien que divergentes sur certaines questions, leurs pensées se complètent et coexistent, littéralement, dans les pages de l'une des revues littéraires les plus estimées de l'époque sans que leur proximité ne pose problème ni n'attire spécialement l'attention des lecteurs. Sauf erreur, il n'y a pas de controverse ni même de désaccord entre les deux écrivains, d'autant que Casgrain, en tant que membre de la direction du périodique, a vraisemblablement pu lire et approuver le «Discours» de son collègue avant sa publication. Malgré tout, l'argumentation déployée par chacun révèle des différences marquantes qui valent d'être relevées.

En ce qui a trait à leur rapport au temps d'abord, les deux auteurs s'entendent pour proposer une vision optimiste de l'avenir. Dans

${ }^{45}$ MLC, p. 30 . 
l'expectative de grandes choses à venir, contrastant avec le chaos du passé, ils commentent l'histoire sur un mode téléologique. Dans les deux cas, l'articulation entre politique et littérature se défait, et les écrits polémiques et revendicateurs d'autrefois paraissent réinterprétés à la lumière d'une orthodoxie nouvelle: Garneau devient notre historien national, Crémazie, notre poète national et AugustinNorbert Morin, un exemple de l'excellente formation dispensée dans le cours classique. Pourtant, la paix sociale acquise dont parle Casgrain peine à se matérialiser dans les textes de son aîné, même en 1866. À l'inverse du bon abbé, Raymond n'a pas la prétention de connaitre l'histoire du pays ni la lente marche du progrès qui permet à son collègue d'annoncer l'avènement du mouvement littéraire en Canada. Il a cependant vécu les révoltes patriotes et sait la précarité qui menace l'identité canadienne-française. Raymond ne se fait pas d'illusion sur «les conquêtes politiques» réalisées par ses contemporains ni, d'ailleurs, sur celles à venir. De son point de vue, le passé n'est pas une manne sacrée, un répertoire presque intarissable d'histoires et de héros méconnus, ainsi que semble le croire Casgrain. La génération présente ne peut se satisfaire des récits de nos glorieuses épopées; il faut encore qu'elle s'en inspire, qu'elle s'en imprègne, car l'histoire doit lui servir de leçon, et pas seulement à redorer le blason du peuple canadien.

Outre le rapport au temps, la religion devient également un point de discorde. De Raymond à Casgrain, et de 1830 à 1866, il y a en effet une mutation importante du lien entre religion et littérature. À de nombreux endroits dans les textes, le mysticisme de l'aîné contraste avec le pragmatisme du cadet, l'attachement au surnaturel s'opposant à la primauté du réel, et la théorie de l'inspiration à celle du reflet. Pour l'historien, la littérature doit être pieuse pour être nationale, simplement parce qu'il s'agit de l'une des spécificités de la population. Il n'y a aucune transcendance ni aucune fonction morale de la littérature qui la détermine dans ce sens, seulement un état de faits, qui, du reste, s'explique «rationnellement» par le positionnement géopolitique du pays, encerclé par 
l'élément anglo-américain. Pour reprendre à nouveau les mots de Micheline Cambron, l'abbé «fait de la littérature une sorte de reflet de la "nature" nationale, un effet identitaire d'où toute distance est évacuée ${ }^{46} »$. Ainsi, la religion est partout présente, mais sert finalement peu l'argumentation du "Mouvement littéraire en Canada", à l'inverse du "Discours sur l'importance des études classiques». Le thème du sacerdoce de l'écrivain romantique, présent dès les premiers textes de Raymond, parcourt bien l'ensemble de son discours sur la littérature. L'éducateur brosse le portrait d'un art bienfaisant, religieux non pas parce qu'il est canadien, mais parce qu'il puise sa source dans la beauté du christianisme. Plus qu'une «expression de la société", cette littérature veut être une école, contribuer à l'édification du peuple, lui proposer des modèles à célébrer, à imiter.

Enfin, l'un des plus importants points de comparaison entre les deux conceptions de la littérature canadienne touche la jeunesse et les recommandations qui lui sont faites par les deux hommes. Ces derniers se rejoignent sur la nécessité des études classiques, qui visent la formation de jeunes gens capables de formuler un discours raisonné et éloquent. Pour le choix de leur carrière, toutefois, l'un prêche la prudence, l'autre l'audace. Joseph-Sabin Raymond ne valorise pas la publicité au même titre que son collègue, n'en témoigne que la somme de ses textes restés inédits, voire sa préférence pour les conférences et les discours, plutôt que pour la publication d'ouvrages sous forme de livres. Il collabore aussi très peu aux journaux, dont il se montre fort critique. Henri-Raymond Casgrain, lui, ne se contente pas de diffuser ses textes, sous toutes les formes possibles, semble-t-il, mais il engage chacune de ses accointances dotées de quelque talent littéraire à faire de même. Des écrivains

${ }_{46}$ Micheline Cambron, «L'asymétrie épistolaire: de l’usage de la correspondance à la conception littéraire chez Octave Crémazie et Henri-Raymond Casgrain", dans Benoît Melançon et Pierre Popovic (dir.), Les facultés des lettres: recherches récentes sur l'épistolaire français et québécois, Montréal, Centre universitaire pour la sociopoétique de l'épistolaire et des correspondances, Université de Montréal, 1993, p. 73. 
comme Crémazie ${ }^{47}$, puis Laure Conan ${ }^{48}$ ont bien connu l'insistance de l'homme de lettres sur ce point. Selon lui, la littérature n'est pas sacrée, mais utilitaire. Elle documente l'histoire et l'avancement intellectuel des peuples, les fait connaître aux autres, les révèle à euxmêmes. La religion n'est pas absente de ce paradigme, mais c'est la Patrie qui est célébrée d'abord, l'Église ensuite.

Romantiques, Raymond et Casgrain le sont tous les deux, même si c'est de façon différente. De 1830 à 1866, le romantisme littéraire canadien change parce que la littérature tant souhaitée commence à prendre forme, à s'autonomiser, à devenir un objet valorisé en soi. La religion n'est pas bien loin, mais la Patrie, la Nation la surpassent peu à peu dans l'ordre des priorités. Il ne suffit plus d'être un écrivain chrétien, il faut encore être un écrivain canadien, et publier, publier beaucoup, en sachant que la publicité sera mal vue par certains. Le rapport au passé, en particulier, reste problématique. Entre ceux qui ont connu les insurrections armées et ceux qui les amalgament aux "deux siècles de luttes incessantes", le fossé se creuse. Néanmoins, il n’y a pas rupture de dialogue; des dialogues de sourds, des angles morts, des contradictions sur lesquelles on préfère ne pas insister, certes, mais aucune rupture, seulement une transition, d'une génération à une autre.

${ }^{47}$ Voir, notamment, Cambron, «L'asymétrie épistolaire», p. 72-93.

${ }^{48}$ Voir, notamment, Nicole Bourbonnais, «Introduction», dans Laure Conan, Angéline de Montbrun, Montréal, Les Presses de l'Université de Montréal, 2007, p. 7-119. Il faut mentionner toutefois que Raymond, comme directeur spirituel de Laure Conan au début des années 1870-1880, l'encourage lui aussi à publier son premier roman, Angéline de Montbrun (1884). Au contraire de Casgrain cependant, il ne possède pas les contacts ou les ressources nécessaires pour lui assurer un contrat d'édition. 